\title{
Wind speed effect on the flow field and heat transfer around a parabolic trough solar collector
}

\author{
A.A. Hachicha, I. Rodríguez, A. Oliva \\ ${ }^{a}$ Heat and mass Transfer Technological Center (CTTC), Universitat Politècnica de \\ Catalunya-BarcelonaTech (UPC) ETSEIAT, Colom 11, 08222 Terrassa (Barcelona), \\ Spain.
}

\begin{abstract}
The parabolic trough solar collector is currently one of the most mature and prominent solar technology for production of electricity. These systems are usually located in an open terrain where strong winds may occur and affect their stability and optical performance, as well as, the heat exchange between the solar receiver and the ambient air. In this context, a wind flow analysis around a parabolic trough solar collector under real working conditions is performed. A numerical aerodynamic and heat transfer study based on Large Eddy Simulations is carried out to characterize the wind loads and the heat transfer coefficients. After the study carried out by the authors in earlier work [? ] at $R e_{W 1}=3.9 \times 10^{5}$, computations are performed at a higher Reynolds number of $R e_{W 2}=1 \times 10^{6}$ and for various pitch angles. The effects of wind speed and pitch angle on the averaged and instantaneous flow have been assessed. The aerodynamic coefficients are calculated around the solar collector and validated with measurements performed in wind tunnel tests. The variation of the heat transfer coefficient around the heat collector
\end{abstract}

\footnotetext{
${ }^{*}$ Corresponding author. Tel.:+34-93-739-8192; fax:34-93-739-8101
} 
element with the Reynolds number is presented and compared to the circular cylinder in cross flow. Unsteady flow is studied for three pitch angles: $\theta=0^{\circ}$, $\theta=45^{\circ}$ and $\theta=90^{\circ}$ and different structures and recirculation regions have been identified. A spectral analysis has been also carried out around the parabola and its receiver in order to detect the most relevant frequencies related to the vortex shedding mechanism which affect the stability of the collector.

Keywords: Parabolic trough solar collector, Wind speed effect, Large Eddy Simulations, Heat transfer coefficient, PTC stability

kinematic viscosity $\left(\mathrm{m}^{2} / \mathrm{s}\right) 02$

pitch angle02

diameter $(m) 02$

frequency $(1 / s) 02$

convective heat transfer coefficient $\left(W / m^{2} K\right) 02$

time $(w / m K) 02$

recirculation length $(m) 02$

temperature $(K) 02$

$\operatorname{velocity}(m / s) 02$

aperture $(m) 02$

computational fluid dynamics 02 
control volume 02

heat collector element 02

heat transfer fluid 02

large eddy simulations 02

million control volume 02

Nusselt number $(h D / k) 02$

parabolic trough solar collector 02

Reynolds-averaged Navier Stokes equations 02

Reynolds number $(U D / \nu) 02$

Strouhal number 02

ambient02

average02

front stagnation point02

glass envelope02

maximum02

minimum02

reference02 


\section{Introduction}

Parabolic trough solar collectors (PTC) are considered as one of the most mature, successful, and proven solar technology for electricity generation. PTCs are typically operated at $400^{\circ} \mathrm{C}$ and a synthetic oil is commonly used as heat transfer fluid (HTF). A PTC consists of a parabolic troughshaped mirror that focus sunrays onto a heat collector element (HCE) that is mounted in the focal line of the parabola. The HTF circulates through the solar field to transport the absorbed heat. The solar field is made up of several solar reflectors composed in series which concentrate the direct solar radiation by means of a Sun-tracking system. The HCE is typically composed of a metal receiver tube and a glass envelope covering it with a vacuum between these two to reduce the convective heat losses.

The thermal and optical performances of PTC are related to the applied load coming from the wind action on the structure and the tracking system. During real work conditions, the array field of solar collectors require a good accuracy in terms of both mechanical strength and optical precision. Such requirements are sensitive to turbulent wind conditions and should be considered in the design of these systems. Hence, a wind flow analysis plays a major role for designing the solar collectors and can lead to a better understanding of the aerodynamic loading around the parabolic reflector, as well as, the convection heat transfer from the HCE.

Since the 1970s, numerous numerical and experimental studies have been proposed to study the heat transfer characteristics of PTC [? ? ? ]. However, wind flow studies around the PTC are scarce. Sandia National Laboratories has published in the early of 1980 some wind tunnel tests [? ? ] to investi- 
gate characteristics of mean wind loads produced by airflow around a PTC. These reports were conducted in different configurations of the PTC and flow field environments. The influence of various geometric design parameters for isolated PTC and for a collector within an array field are assessed. More recently, series of wind tunnel experiments were conducted by ? ] from March 2001 to August 2003. This wind-tunnel study includes the distribution of local pressure across the face of the solar collector using a 1:45 model. Two versions of instrumented collector models were used to measure the loads and pressure distribution across the face of the collector. One was a lightweight model for measuring wind loads using a high-frequency force balance and the other was a pressure-tapped model designed to obtain the pressure distribution across the face of the collector. The effect of the PTC position in an array of solar collectors was also examined.

The majority of the numerical studies for studying the wind flow around solar collectors are based on the Reynolds-Averaged Navier Stokes equations (RANS) [? ? ] which suffer from inaccuracies in predictions of flow with massive separations.

A recent study by the authors [? ] based on Large Eddy Simulations (LES) allowed to quantify the fluid flow and heat transfer around a PTC for various pitch angles and a fixed wind speed $1 \mathrm{~m} / \mathrm{s}$. The study showed that this kind of detailed numerical simulations are feasible, but the effects of a higher wind speed was not explored.

In the present work, following the previous experiences, the impact of wind speed closer to real working conditions is considered. The independence of the drag and lift coefficients is studied to confirm the hypotesis made by the 
experimental measurements in wind tunnels of down-scaled PTC prototypes ? ]. The natural frequencies around the PTC structure and the HCE are also studied. To do this, the wind flow around the PTC at a wind speed of 3 $\mathrm{m} / \mathrm{s}$ is studied and compared to the results obtained at $1 \mathrm{~m} / \mathrm{s}$. These cases correspond with Reynolds numbers of $R e_{W 1}=3.6 \times 10^{5}$ and $R e_{W 2}=1 \times 10^{6}$ (the Reynolds number is defined in terms of the free-stream velocity and the

aperture $R e_{W}=\frac{U_{r e f} W}{\nu}$ ). Wind speed effects on unsteady and averaged fluid flow and heat transfer characteristics are assessed. Furthermore, a power spectra analysis is carried out to analyse the impact of the unsteady flow conditions on the PTC stability.

\section{PTC numerical model}

\subsection{Mathematical model}

The same methodology presented in the previous work [? ] for solving the fluid flow and heat transfer around the PTC is here adopted.The CFD\&HT code Termofluids [? ] has been used to simulate the complex fluid dynamics around the solar collector and its receiver by means of Large-Eddy simulations (LES).

\subsection{Definition of the case. Geometry and boundary conditions}

Large-eddy simulations of the wind flow around a PTC at $R e_{W}=10^{6}$ and different pitch angles of $\left(\theta=0^{\circ}, 45^{\circ}, 90^{\circ}, 135^{\circ}\right.$ and $\left.270^{\circ}\right)$ have been performed. Here, Reynolds number has been defined in terms of the free-stream velocity $U_{\text {ref }}$ and the parabola aperture $W\left(R e_{W}=\frac{U_{r e f} W}{\nu}\right)$. This Reynolds number corresponds with a wind speed of $U_{r e f}=3 \mathrm{~m} / \mathrm{s}$ which is a typical value of 
wind speed encountered in solar plants. In addition, the flow around the parabola has been compared to that obtained by the authors at a lower Reynolds numbers of $R e=3.6 \times 10^{5}\left(U_{\text {ref }}=1 \mathrm{~m} / \mathrm{s}\right)$ [? ].

All computed flows are around a full-scale Eurotrough solar collector [? ] and its typical HCE with a stainless steel absorber (inner/outer diameter of 6.6/7.0 cm) and glass cover (10.9/11.5 cm of inner/outer diameter). As in the previous work [? ], the same domain size of $25 W \times 9 W \times \pi W$ with the same steam-, cross stream- and span-wise directions has been used. The parabola of aperture $W=5.8 \mathrm{~m}$ is located at $5 \mathrm{~W}$ in the stream-wise direction (see figure ??). For solving the computational domain, no-slip conditions at the parabola and HCE have been imposed. At the inlet, a uniform inlet velocity profile has been prescribed. For the top and bottom boundaries, slip conditions have been set, whereas in the span-wise direction, the flow has been considered to be spatially periodic, thus periodic boundary conditions have been imposed. For solving the energy equation, temperatures of the glass cover and ambient air are fixed to $T_{g}=350 \mathrm{~K}$ and $T_{a m b}=300 \mathrm{~K}$, respectively. A Neumann boundary condition $\left(\frac{\partial T}{\partial n}=0\right)$ is prescribed in the top, bottom and outlet boundaries for temperature.

For more details about boundary conditions, the reader is referred to [? ]. In Termofluids, the governing equations have been discretised on a collocated unstructured grid arrangement by means of finite volume techniques using second-order conservative schemes [? ]. The 3D meshes used for solving the computational domain have been obtained by the constant-step extrusion in the span-wise direction of a two-dimensional unstructured grid. Although not shown here, extensive grid refinements for each pitch angle have been 
Table 1: Details of adopted meshes for each pitch angle

\begin{tabular}{lllllll}
\hline Position & $0^{\circ}$ & $45^{\circ}$ & $90^{\circ}$ & $135^{\circ}$ & $180^{\circ}$ & $270^{\circ}$ \\
Mesh plane & 112322 & 104477 & 102914 & 99281 & 106223 & 104188 \\
Number of planes & 96 & 96 & 96 & 96 & 96 & 96 \\
Grid size(MCVs) & 10.78 & 10.02 & 9.87 & 9.53 & 10.19 & 10.0 \\
\hline
\end{tabular}

conducted. Details of the final computational meshes for each pitch angle are given in table ??.

\section{Heat transfer from a circular cylinder in cross flow and wind speed effects}

In order to analyse the influence of the wind speed in the heat transfer of the HCE, the numerical model has been first applied on a circular cylinder in cross flow. In this work, simulations have been performed for a Reynolds number of $R e_{D}=21600$ (here Reynolds number is defined in terms of the free-stream velocity and cylinder diameter, $R e_{D}=U_{r e f} D / \nu$ ) which corresponds with a wind speed of $3 \mathrm{~m} / \mathrm{s}$. Heat transfer characteristics around the cylinder have been calculated and compared against experimental measurements of ? ]. In addition, results have also been compared to the lower Reynolds number of $R e=7200$ [? ] (which corresponds with wind speed of $1 \mathrm{~m} / \mathrm{s}$ ). The boundary conditions and mesh distribution have been considered in a similar way as the previous section ??. The computational domain is extended to $[-15 D, 25 D] ;[-10 D, 10 D] ;[0, \pi D]$ in the stream-, crossand span-wise directions respectively, and the cylinder with a diameter $D$ is 
placed at $(0,0,0)$. The results shown herein are computed for a finer grid of $147000 \times 64$ planes (i.e. $147000 \mathrm{CVs}$ in the 2D planes extruded in 64 planes yielding about 9.4 MCVs).

In figure ??, the predicted local Nusselt number around the circular cylinder is plotted. For comparison, the results of ? ] are also shown. As can be observed a fair agreement between both numerical and experimental results has been obtained. In general, numerical results follow the same trend that the experimental ones. The minimum local values of the heat transfer coefficient occurs at about $85^{\circ}$ from the stagnation point, whereas the maximum values are reached at the stagnation point and at the rear end of the cylinder. As observed in $R e_{D}=7200$ (see [? ]), large differences from experiments are found to occur in the back size of the cylinder as the flow fluctuations are the largest which makes more difficult experimental measurements [? ]. When comparing both Reynolds numbers (see figure ??), i.e, $R e_{D}=7200$ and $R e_{D}=21600$, it can be observed the increasing of inertial effects due to the increasing of Reynolds number which lead to the earlier separation of the boundary layer. Indeed, there is a displacement of the location of the minimum Nusselt number at $R e_{D}=21600$ towards the stagnation point. The variation of the Nusselt number in the rear zone of the cylinder is smoother at $R e_{D}=7200$ and a secondary peak is observed for $R e_{D}=21600$ (at about $118^{\circ}$ ). By increasing the Reynolds number from $R e_{D}=7200$ to $R e_{D}=21600$, the overall magnitude of the Nusselt number increases by a factor of 2 from 52.2 to 101.1 The value reported on the experiments was 103.4 (for $R e_{D}=21600$ ), being the average difference between 
both numerical and experimental results of about $2.2 \%$.

\section{Results and discussion}

As aforementioned, simulations have been conducted to study the wind effects around the PTC for two Reynolds numbers based on the aperture $R e_{W 1}=3.6 \times 10^{5}$ and $R e_{W 2}=1 \times 10^{6}$ and different pitch angles of $\theta=$

$0^{\circ}, \theta=45^{\circ}, \theta=90^{\circ}, \theta=135^{\circ}, \theta=180^{\circ}, \theta=270^{\circ}$. Thereafter, these effects are analysed in terms of the average forces on the parabola, the flow configurations and the instantaneous flow.

\subsubsection{Wind speed effects on the averaged forces}

The average forces on the parabola have been validated respect to the experimental data [? ] in the previous work [? ]. To that end, the wind flow was studied around a typical LS-2 parabolic trough solar collector (without solar receiver) as proposed in the experimental study [5] and simulations were performed for a full-scale case with a Reynolds number about $2 \times 10^{6}$. In the present work, drag and lift coefficients have been computed for the PTC under study at different pitch angles and for both Reynolds numbers. The comparison with experimental measurements and those obtained for the LS-2 PTC are depicted in figure ??. As can be seen from figure ??, numerical results obtained are almost within the error-bars of experimental measurements from the wind-tunnel data [? ]. Discrepancies between computed and measured aerodynamic coefficients are mainly due to the unsteady flow behaviour and ground effects, which may affect the flow structures and separations behind the PTC and requires long measurement duration. The averaged aerodynamic coefficients at both Reynolds numbers exhibit an almost 
identical profile, which proves the stability of the aerodynamic coefficients at this range of Reynolds number. The predicted results are also in agreement with the experimental observations of ? ]. In their scaled-down experimental tests carried out at Reynolds numbers $R e<5 \times 10^{4}$, they concluded that beyond $R e=5 \times 10^{4}$ load coefficients were independent of the Reynolds numbers, being thus directly extrapolated to a full-scale PTC. In the light of the load coefficients here presented, the aforementioned hypothesis of ? ] can be confirmed. It is also worth noting that the independence of averaged aerodynamic coefficients with Reynolds numbers is also commented in other experimental results [? ]. However, it was mentioned that it could be affected where the leading edge is close to the alignment with the stream causing some errors on the lift coefficient [? ].

Yet, there are also some differences between numerical results of the LS2 PTC and Eurotrough PTC which are due to the geometry of both solar collectors. It should be pointed out that from a numerical point of view the results presented for the LS-2 are statistically more converged in time than those for the Eurotrough. This is due to the complex grid used in the simulation of the Eurotrough PTC. In the latter, the geometry considered also included the receiver tube (which was not included in the LS-2 simulations), which imposes a large difference in scales between the parabola and the receiver. Thus, in the numerical simulations the explicit algorithm requires smaller time-steps (of about $5 \times 10^{-5}$ ) for solving all the relevant temporal scales of the flow, dominated by the flow around the receiver. This decrease in the time-step, together with the more complex flow around the receiver impose larger simulation time in order to reach a statistical averaged flow. 


\subsubsection{Wind speed effects on the averaged flow}

The time-averaged flow is also studied for different pitch angles and compared for both Reynolds numbers. Different structures and recirculation regions are encountered around the collector and the HCE. These flow structures are strongly related to the collector orientation and the pitch angle. The effect of the wind speed on the structures and recirculation regions observed around the PTC has also been assessed. By increasing the Reynolds number, the flow pattern does not change and the recirculation regions are similar to those found at $R e_{W}=3.6 \times 10^{5}$ with small variation of the recirculation length behind the parabola as shown in figure ??. The recirculation length for different pitch angles and for both Reynolds number is determined and presented in table ??. It can be seen from this table that the recirculation length is almost in the same range for both Reynolds numbers. This similarity has also been depicted in the comparison of averaged streamlines for both Reynolds numbers (see figure ??). However, in general terms it is observed that the recirculation length enlarges with the Reynolds number when the concave surface of the parabola is exposed to the wind direction, i.e $\theta<90^{\circ}$, and shrinks for the convex surface configuration $\left(\theta>90^{\circ}\right)$.

At vertical position of $\theta=0^{\circ}$, a large recirculation region is observed behind the PTC with a maximum drag and minimum lift forces. This region increases with the Reynolds number and extends up to $8.32 \mathrm{~W}$ at $R e_{W 2}=1 \times 10^{6}$. By moving the PTC to a pitch angle of $45^{\circ}$, the recirculation decreases compared to the vertical position and the shear layer is reduced. However, its length at $R e_{W 2}$ is almost a $30 \%$ larger than for $R e_{W 1}$ extending up to $3.06 \mathrm{~W}$. The drag coefficient decreases whereas the absolute 
Table 2: Variation of the ratio of the non-dimensional recirculation length $\left(L_{r} / W\right)$ to collector aperture with pitch angle and comparison between both Reynolds numbers

\begin{tabular}{lllllll}
\hline Pitch angle & $\theta=0^{\circ}$ & $\theta=45^{\circ}$ & $\theta=90^{\circ}$ & $\theta=135^{\circ}$ & $\theta=180^{\circ}$ & $\theta=270^{\circ}$ \\
\hline$R e_{W 1}=3.6 \times 10^{5}$ & 7.65 & 2.21 & 0.09 & 1.6 & 9 & 0.2 \\
$R e_{W 2}=1 \times 10^{6}$ & 8.32 & 3.06 & 0.1 & 1.47 & 8.3 & 0.17 \\
\hline
\end{tabular}

value of the lift coefficient increases.

The minimum of recirculation length occurs at the horizontal position of $90^{\circ}$, where only small eddies are encountered in the leeward side of the PTC. This value is about $0.1 \mathrm{~W}$ for both Reynolds numbers. The drag forces also reach their minimum values at this position. At a pitch angle of $135^{\circ}$, the recirculation region enlarges again and a pair of medium-sized eddies are formed behind the PTC where negative pressure is observed. However, due to the aerodynamic profile of the collector, the recirculation length shrinks with Reynolds number and extends up to $1.47 \mathrm{~W}$ at $R e_{W 2}$. By moving the PTC to the vertical position at $180^{\circ}$, the recirculation length reaches a new maximum and similar to $\theta=0^{\circ}$ two large eddies are formed behind the PTC. The shear layer is again elevated and drag forces are also increased. Comparing both Reynolds numbers at this pitch angle, the recirculation length is about 7\% smaller for the higher Reynolds number which is due to the convex surface configuration. When the PTC is placed at the stow position, i.e $\theta=270^{\circ}$, the recirculation region is sharply reduced similarly to the working position $\theta=90^{\circ}$. Therefore, the drag forces decrease as well. At this po- 
sition, the recirculation length remain almost unchanged with the Reynolds number around $0.2 \mathrm{~W}$.

\subsubsection{Wind speed effects on the heat transfer around the HCE}

As it has been discussed in the previous work [? ], the convection taking place around the HCE is divided into forced convection (for pitch angles of $\theta=0^{\circ}, 45^{\circ}, 90^{\circ}$ and $270^{\circ}$ ) and mixed (free and forced) convection (for pitch angles of $135^{\circ}$ and $180^{\circ}$ ). In figure ??, the distribution of the local Nusselt number for different pitch angles together with the comparison between both Reynolds numbers is shown. According to this figure, the profile of Nusselt number around the HCE is affected with the pitch angle and the displacement of the fluid structure around the HCE due to the tilt of the parabola. At higher wind speed, i.e. higher Reynolds numbers, the profile of Nusselt

number follows a similar trend to that observed at the lower speed $R e_{W 1}$. However, there is an increase in the magnitude and the peaks become more pronounced at all pitch angles. Moreover, the effect of the parabola and the ground becomes more significant with increasing the Reynolds number. While at the lower Reynolds number the local distribution of the Nusselt number was observed to follow the same trend to that of a circular cylinder in cross flow [? ], this is not the case for $R e_{W 2}$. This can be clearly observed in figure ??, where the distribution at pitch angles of $\theta=90^{\circ}$ and $\theta=270^{\circ}$ (working and stow modes), together with the circular cylinder in cross-flow are depicted. Large differences in the behaviour are obtained in the rear zone. At these positions, the combined effect of the parabola and the ground tend to reduce the large fluctuations of the near wake leading to a smoother 
distribution of the Nusselt number in the rear zone especially when it comes to the minimum and maximum magnitudes.

Table ?? summarizes the average, front stagnation, maximum and minimum Nusselt numbers $\left(N u_{\text {avg }}, N u_{f s p}, N u_{\max }, N u_{\min }\right.$, respectively) together with the location where the extrema occurs for both Reynolds number $R e_{W 1}$ and $R e_{W 2}$. These results are also compared with available experimental data [? ] and the correlation of Zukauskas [? ], for a circular cylinder and the numerical results presented in previous work [? ].

As expected, the higher the Reynolds number, the higher the average magnitude of the Nusselt number. Furthermore, compared to the circular cylinder in cross flow at the same Reynolds number there is a reduction in the average Nusselt number. This is mainly due to the effect of the parabola which is desirable as it reduces the heat losses from the HCE, thus improving the performance of the PTC. When the parabola is placed at the vertical position of $\theta=0^{\circ}$, the averaged Nusselt number is increased by $70 \%$ compared to the lower wind speed case and reduced to $59 \%$ compared to the circular cylinder in cross flow case. At a pitch angle of $\theta=45^{\circ}$, the averaged Nusselt number is increased by $68 \%$ compared to the lower wind speed case and reduced to $40 \%$ compared to the circular cylinder in cross flow case. Although the effect of the parabola is less important than the vertical position $\theta=0^{\circ}$, the peaks for a pitch angle of $45^{\circ}$ increase considerably at high wind speed (see also figure ??-b). The profile of Nusselt number still follows the tilt of the parabola and remains unchanged with increasing the Reynolds number. The distribution of the Nusselt number at the working position $\theta=90^{\circ}$ is symmetric (see figure ??-c) and its averaged magnitude increased 
by $85 \%$ when compared to the lower wind speed case. At a pitch angle of $\theta=135^{\circ}$, the averaged Nusselt number is increased by $72 \%$ compared to the lower wind speed case and reduced to $55 \%$ compared to the circular cylinder in cross flow case. At this position mixed convection occurs and the profile of Nusselt number is quite different to that of the circular cylinder in cross flow. The effect of wind speed is significant and the peaks increase sharply compared to the lower wind speed which exhibits a flatter profile (see figure ??-d). A similar behaviour takes also place at $\theta=180^{\circ}$ where mixed convection occurs. The averaged Nusselt number at $R e_{W_{2}}$ is a $61 \%$ higher than at $R e_{W 1}$ and about $64 \%$ compared to the circular cylinder. By moving the PTC to the stow position $\theta=270^{\circ}$, the Nusselt number profile is also symmetric (see figure ??-f) and similar to the working position $\theta=90^{\circ}$. The averaged Nusselt number is a $62 \%$ higher at $R e_{W 2}$ compared to the lower wind speed case and $30.5 \%$ compared to the circular cylinder. 


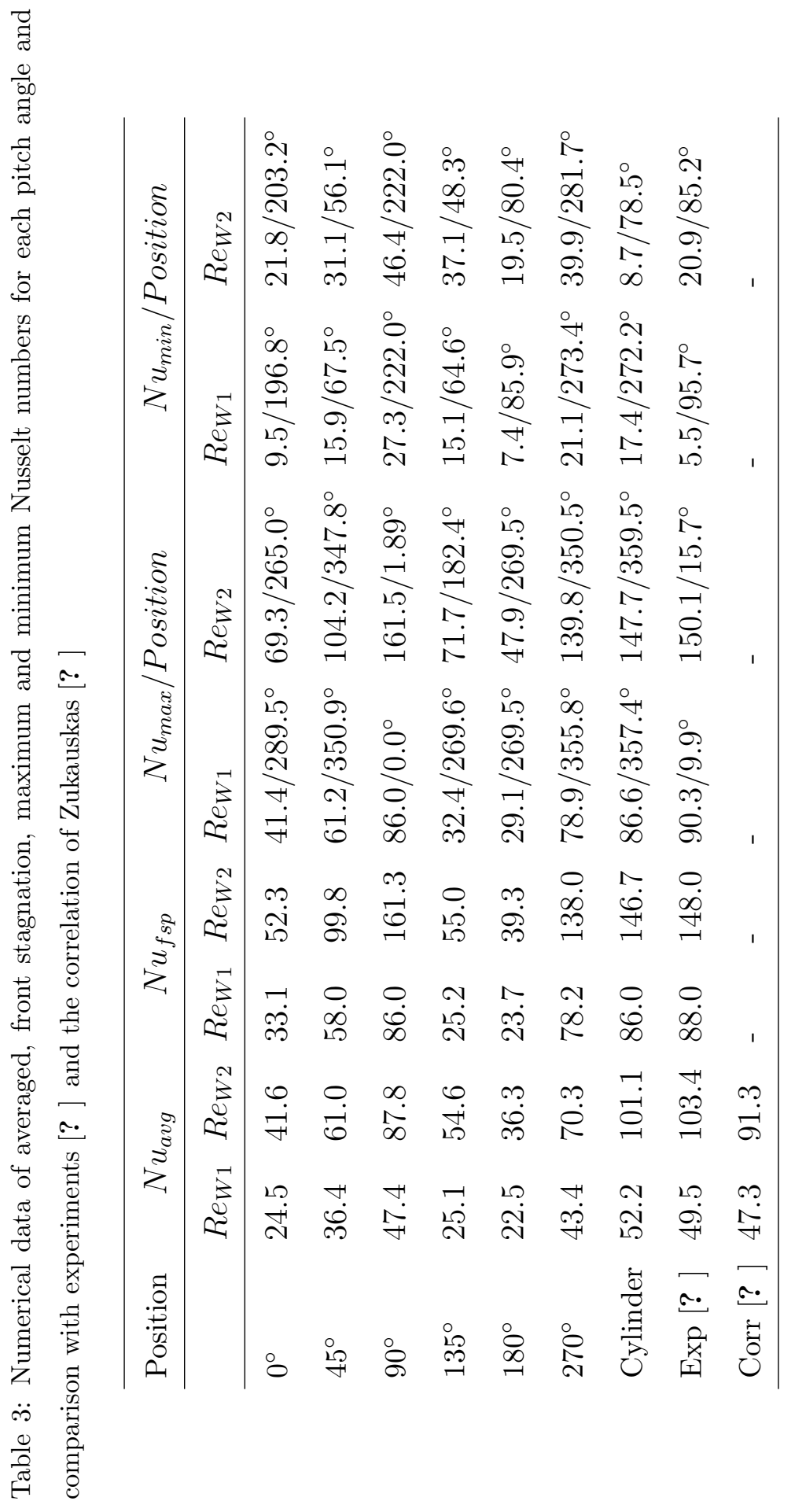




\subsection{Wind speed effects on the unsteady flow}

The study of the unsteady flow field around the PTC at different pitch angles might be useful to gain insight into the flow behaviour in order to be able of controlling undesirable fluctuating forces. It should be borne in mind that the collector structure should sustain wind loads as well as keep accurate sun tracking. Vortex shedding in the wake of the PTC induces alternating forces perpendicular to the wind direction which might affect its structure. These vortices, depending on the pitch angle, are shed at a determined fre-

quency and might produce undesirable effects such as deflections, vibrations, torsional moments, resonance with the structure and, at the end, stresses leading to the structure failure. Thus, in order to study the unsteady behaviour, instantaneous flow structures and frequencies have been examined at three pitch angles of $\theta=0^{\circ}, \theta=45^{\circ}$ and $\theta=90^{\circ}$. These angles describe the three possible positions occupied by the parabola, i.e. vertical, inclined and horizontal positions.

Single-point measurements have been carried out by positioning probes at different locations around the parabola. The frequencies of the fluctuations of the cross-stream velocity component have been computed by using the Lomb periodogram technique [? ] and the resulting spectra have been averaged in the periodic direction. For the sake of brevity only 3 probes for each pitch angle are shown and compared for both Reynolds numbers. Only the most relevant results are here presented. The location of these probes are given in figure ??.

For all pitch angles, and due to the sharp edges of the parabola the fluid undergoes a rapid transition to turbulence. Depending on the pitch angle, 
the sharp edges produce flow separation which prevents the pressure from recovering (large recirculation region behind the parabola) and therefore, a high pressure drag is observed. The separated flow at the sharp edges forms a shear-layer which resembles to be much like that formed behind a square cylinder or a normal plate [? ]. These shear layers are characterised by the formation of instabilities which cause the fluid to become unstable in the presence of sharp corners. These instabilities increase in amplitude and accumulate into large vortical structures which are shed into the wake. As it will be further explained, depending on the pitch angle the level of coherence of these structures might form a turbulent wake similar to a von Kármán vortex street.

In figure ?? the velocity flow field of the three pitch angles and both Reynolds numbers $\left(R e_{W 1}\right.$ and $\left.R e_{W 2}\right)$ are depicted. Qualitatively, the instantaneous flow field are quite similar for both Reynolds numbers. A large separated zone is observed at $\theta=0^{\circ}$. The turbulent flow in the detached region produces a large depression region in the back of the PTC being the responsible for the large value of the drag coefficient obtained. The shear layer is more elevated at this position and the flow seems to follow the curvature of the PTC. However, the height of the recirculation zone decreases as the Reynolds number increases and the flow becomes more turbulent. Similar to previous observations [? ], the height of the detached flow tends to decrease as the pitch angle moves from $\theta=0^{\circ}$ to $\theta=90^{\circ}$. The latter is the most favourable position for the PTC to work in terms of both unsteady forces and magnitude of averaged aerodynamic coefficients.

The structures formed at the different pitch angles are also observed by 
means of the instantaneous pressure map (see figure ??). A striking fact is that depending on the pitch angle the wake structure is completely different.

Starting from $\theta=0^{\circ}$, shear-layer instabilities at both sides of the parabola are observed (see figure ??a). These structures grow-up, but as a consequence of the interaction of the bottom shear-layer with the ground, the transverse motion of the separated shear layers is suppressed with the formation of vortices flowing downstream in a parallel manner. As a result, the level of coherence of the flow is low and only a small peak in the spectrum of the cross-stream velocity fluctuations is observed. This peak is captured at probe P2 (see figure ??) for $R e_{W 2}$ at $S t_{W}=\frac{f W}{U_{r e f}}=0.34$. The peak is more pronounced at the higher Reynolds number than for the lower one but still it can be seen at $S t_{W}=0.28$ as a small footprint in the energy for $R e_{W 1}$.

At $\theta=45^{\circ}$, as the bottom corner moves off the ground, both shearlayers are allowed to interact and vortices shed into the wake form a von Kármán-like vortex street (see figure ??b). By analysing the energy spectrum for cross-stream velocity fluctuations of probe P1 (see figure ??), one can notice that for the higher Reynolds number the peak in the energy is more distinguishable indicating a high coherence in the signal. In fact, the signal capture what can be identified as a double-peak mechanism. As the process of vortex shedding is asymmetric vortices formed at the top corner have a slight different period than those formed at the bottom corner leading to the double-peak observed in the energy spectrum. The same double-peak is also captured at the lower Reynolds number but at a lower frequency. Note also that at the lower Reynolds number, the flow is not so coherent and turbulent fluctuations are less energetic. 
Finally, when the parabola is at $\theta=90^{\circ}$, leading-edge corner shear-layers instabilities move downstream and interacts with those structures formed in the wake of the receiver, breaking down into more complicated and disorganised structures near the trailing-edge corner (see figure ??c). As a result, the energy spectrum around the PTC at this position can not capture a distinguishable peak corresponding to the vortex shedding phenomenon (see figure ??).

For all pitch angles, the observed frequencies are better captured for the high Reynolds number where the energy peak is more pronounced. From the stability point of view of the PTC, even though the magnitude of the drag forces at vertical positions are higher, turbulence fluctuations are more important at intermediate positions $\left(0^{\circ}<\theta<90^{\circ}\right)$. At these positions, the interaction between the shear-layer formed at both corners of the parabola produces an unsteady flow with a highly coherent vortex shedding which might lead to vibrations and the horizontal position is also demonstrated to be the most favourable position as it presents the minimum drag forces and turbulence fluctuations.

In addition, the spectral analysis is also carried out around the HCE to detect the relevant frequencies related to the receiver tube. Depending on the pitch angle, vortex shedding behind the HCE is also detected (see figure ?? ). Similar to the parabola, it is better captured at the higher Reynolds number but less coherent than the signal captured in the flow past a circular cylinder (see for instance [? ] ). This is due to the interaction of the flow with the parabola and to the turbulent fluctuations that this interaction produces which might be seen as broaden peaks (see for instance figure ??b,c) being 
the energy distributed along a large range of frequencies.

In spite of this, vortex shedding is captured and the results show that as the pitch angle increases from $0^{\circ}$ to $90^{\circ}$ the vortex shedding frequency increases and approaches to the typical value encountered in circular cylinder $S t_{D}=\frac{f D}{U_{r e f}}=0.2[? ?]$. Indeed, the value of Strouhal number turns from $S t_{D}=0.05$ at pitch angle $\theta=0^{\circ}$ to $S t_{D}=0.19$ at pitch angle $\theta=90^{\circ}$.

This can be explained by the effect of the parabola on the HCE which decreases by moving to the horizontal position. It should be pointed out that the unsteady flow and spectral analysis presented in this work for three pitch angles, i.e. $0^{\circ}, 45^{\circ}$ and $90^{\circ}$ can be extrapolated to the other positions because of the similarity of the flow configuration.

\section{Conclusions}

In the present work, a numerical study based on LES of the fluid flow and heat transfer around a parabolic trough solar collector and its receiver tube has been performed. The effects of wind speed and pitch angle on the aerodynamic behaviour and heat transfer characteristics around the PTC at Reynolds numbers similar to that encountered in working conditions have been addressed. It has been concluded that the averaged aerodynamic coefficient are stable with the Reynolds numbers in conformity with experimental results from literature. Furthermore, the structures and recirculation regions observed in the time-averaged flow around the PTC and the HCE are quite

similar for the Reynolds numbers studied. However, a small variation of the recirculation length behind the parabola has been identified due to the aerodynamic profile and depending to the collector orientation. Heat transfer 
coefficients around the HCE are also calculated and compared for different pitch angles and wind speeds. The distribution of Nusselt number for the higher wind speed shows a similar trend to the lower wind speed with higher magnitude and significant peaks. By studying the unsteady flow around the PTC, undesirable effects on the stability of the collector have been addressed for different pitch angles. Indeed, instantaneous flow structures and frequencies have been studied and compared for different orientations and Reynolds numbers. It has been observed that the turbulence is incoherent in the vertical position and becomes much more coherent by moving to intermediate positions allowing the interaction between upper and lower shear layers. This interaction is the consequence of the formation of a von Kármán-like vortex street and has been clearly detected in different stations. In general, the observed frequencies around the PTC are better captured at high Reynolds number and turbulence fluctuations are more important at inclined position. As a result, care must be taken when operating the collector at these positions, specially under high wind loads, as these turbulent fluctuations might be responsible for vibrations and stresses which lead to structure failure. Similar to the parabola, vortex shedding frequency has also been detected behind the HCE. This frequency varies with the pitch angle and approaches to the typical value encountered in circular cylinder when the parabola is placed at horizontal position.

\section{Acknowledgements}

This work has been partially financially supported by the spanish "Ministerio de Economia y Competitividad, Secretaria de Estado de Investigación, 
Desarrollo e Innovación", through project ENE2010-17801. A.A. Hachicha also wishes to thank "Agencia Española de Cooperación Internacional para el Desarrollo (AECID)" for its support in the form of a doctoral scholarship.

\section{References}

[] Hachicha A, Rodríguez I, Castro J, Oliva A. Numerical simulation of wind flow around a parabolic trough solar collector. Applied Energy 2013;107:426-37.

[] Hachicha A, Rodríguez I, Capdevila R, Oliva A. Heat transfer analysis and numerical simulation of a parabolic trough solar collector. Applied Energy 2013;111:581-92.

[] You C, Zhang W, Yin Z. Modelling of fluid flow and heat transfer in a trough solar collector. Applied Thermal Engineering 2013;54(1):247-54.

[] Silva R, Pérez M, Fernández-Garcia A. Modelling and co-simulation of a parabolic trough solar plant for industrial process heat. Applied Energy 2013;106:287-300.

[] Peterka J, Derickson R. Wind load design methods for ground-based heliostats and parabolic dish collectors. Tech. Rep. SAND 92-7009; Sandia National Laboratories; 1992.

[] Randall D, McBride D, Tate R. Parabolic trough solar collector wind loading. In American Society of Mechanical Engineers, Energy technology conference and exhibition 1980;1:18-20. 
[] Hosoya N, Peterka J.A.and Gee R, Kearney D. Wind tunnel tests of parabolic trough solar collectors. Tech. Rep. NREL/SR-550-32282; National Renewable Energy Laboratory; 2008.

[] Naeeni N, Yaghoubi M. Analysis of wind flow around a parabolic collector (1) fluid flow. Renewable Energy 2007;32(11):1898-916.

[] Zemler M, Bohl G, Rios O, Boetcher S. Numercial study of wind forces on parabolic solar collectors. Renewable Energy 2013;60:498-505.

[] Lehmkuhl O, Perez-Segarra CD, Borrell R, Soria M, Oliva A. TERMOFLUIDS: A new Parallel unstructured CFD code for the simulation of turbulent industrial problems on low cost PC Cluster. In: Tuncer I, Gülcat U, Emerson D, Matsuno K, editors. Proceedings of the 2005 Parallel Computational Fluid Dynamics Conference; vol. 67 of Lecture Notes in Computational Science and Engineering. Springer; 2007, p. $275-82$.

[] Geyer M, Lüpfert E, Osuna R, Esteban A, Schiel A, Zarza E, et al. Eurotrough-parabolic trough collector developed for cost efficient solar power generation. In: 11th International Symposium on Concentrating Solar Power and Chemical Energy Technologies. Zurich, Switzerland; 2002 .

[] Verstappen R, Veldman A. Symmetry-preserving discretization of turbulent flow. Journal of Computational Physics 2003;187:343-68.

[] Scholten J, Murray D. Unsteady heat transfer and velocity of a cylinder 
in cross flow-i. low freestream turbulence. Int Journal of Heat and Mass Transfer 1998;41(10):1139-48.

[] Zukauskas A. Heat transfer from tubes in cross flow. Advances in Heat Transfer 1972;8:93-160.

[] Lomb N. Least-squares frequency analysis of unequally spaced data. Astrophysics and Space Science 1976;39:117-462.

[] Gerrard J. The wakes of cylindrical bluff bodies at low Reynolds number. Philosophical Transactions for the Royal Society of London 1978;288(A):351-82.

[] Lehmkuhl O, Rodríguez I, Borrell R, Oliva A. Low-frequency unsteadiness in the vortex formation region of a circular cylinder. Physics of Fluids 2013;25(085109). Doi:10.1063/1.4818641.

[] Fey U, König M, Eckelmann H. A new Strouhal-Reynolds-number relationship for the circular cylinder in the range $47<\operatorname{Re}<2 \times 10^{5}$. Physics of Fluids 1998;10(7):1547-9.

[] Zdravkovich M. Flow around circular cylinders; vol. 2. Oxford University Press; 2003. 